\title{
Estudo de ideação suicida em adolescentes de 15 a 19 anos
}

\author{
Vivian Roxo Borges \\ Universidade Regional Integrada do Alto Uruguai e das Missões \\ Instituição Educacional São Judas Tadeu \\ Blanca Susana Guevara Werlang \\ Pontifícia Universidade Católica do Rio Grande do Sul
}

\begin{abstract}
Resumo
Este estudo objetivou identificar a presença de ideação suicida em adolescentes da população geral, da cidade de Porto Alegre, com idades entre 15 e 19 anos; caracterizar os adolescentes com e sem ideação suicida quanto à intensidade de depressão/desesperança; identificar, nos adolescentes com ideação suicida, o nível de associação entre ideação suicida e depressão/desesperança e identificar, nos adolescentes com ideação suicida, quais variáveis foram encontradas como mais associadas à ideação suicida. Utilizou-se uma ficha de dados sóciodemográficos, Escala de Ideação Suicida de Beck, Inventário de Depressão de Beck e Escala de Desesperança de Beck. Os dados foram analisados através do cálculo de freqüências, pelo teste qui-quadrado e regressão logística. Dos 526 adolescentes da amostra, 36\% apresentaram ideação suicida. Destes, 36\% apresentaram sintomas de depressão e $28,6 \%$ de desesperança (moderada e/ou grave). As variáveis mais associadas à ideação suicida foram: sexo feminino, tentativa de suicídio de amigo, depressão e desesperança.
\end{abstract}

Palavras-chave: ideação suicida; adolescência; depressão; desesperança

\begin{abstract}
Study of suicide ideation in adolescents from 15 to 19 years old. This study aimed at identifying suicide ideation among adolescents aged 15 to 19 years old within the general population of Porto Alegre city, Brazil; characterizing adolescents with and without suicide ideation as to depression/hopelessness intensity; identifying suicide ideation, association level between suicide ideation and depression/hopelessness in those youngsters; as well as identifying the variables most associated to suicide ideation in those adolescents showing this behavior. A social-demographic datasheet, Beck Scale for Suicide Ideation, Beck Depression Inventory and Beck Hopelessness Scale were used. Data were submitted to frequency calculation, chi-square test and logistic regression analysis. From the 526 adolescents in the sample, 36\% presented suicide ideation. Out of them, $36 \%$ showed depression symptoms and 28,6\% hopelessness (moderate and/or severe). The variables most associated to suicide ideation were: feminine gender, a friend's suicide attempt, depression and hopelessness.
\end{abstract}

Keywords: suicide ideation; adolescence; depression; hopelessness

$\mathrm{V}$ ários estudos têm revelado dados epidemiológicos que demonstram um aumento na incidência de suicídio na população geral em todo o mundo. No que diz respeito a adolescentes, este dado vem sendo considerado como a terceira causa de morte, também, em termos mundiais (Bertolote \& Fleischmann, 2004; De Leo, Bertolote, \& Lester, 2003; Goldman \& Beardslee, 1999; Mann, 2002; Maris, Berman, \& Silverman, 2000; Shaffer \& Pfeffer, 2001; WHO, 2001).

No Brasil, os índices de suicídio nas diversas faixas etárias não são tão alarmantes como em países da Europa oriental (Estônia, Letônia, Lituânia, Finlândia, Hungria, Rússia) e da Ásia (China e Japão), mas, em relação à adolescência, como ocorre na maioria dos países mencionados, as taxas de suicídio vêm, também, aumentando (De Leo, 2004; WHO, 2001).
Um estudo publicado por Souza, Minayo e Malaquias (2002) demonstrou que Porto Alegre e Curitiba são consideradas as capitais com os maiores índices de suicídio registrados em jovens com idades entre 15 e 24 anos, comparadas com outras nove capitais (Belém, Fortaleza, Natal, Recife, Salvador, Belo Horizonte, Vitória, Rio de Janeiro e São Paulo). Este dado torna-se alarmante principalmente porque as estatísticas sobre suicídio são falhas e subestimadas, ainda mais no que se refere a adolescentes, sendo que seus atos autodestrutivos são, muitas vezes, negados e escondidos pela família.

O período da adolescência pode ser considerado, muitas vezes, como um momento intenso, de conflitos e mudanças. Na busca de uma solução para seus problemas, estes jovens podem, por exemplo, recorrer a comportamentos agressivos, 
impulsivos ou suicidas. A partir disso, a ideação suicida pode ser considerada como um primeiro passo para um eventual suicídio (Barrios, Everett, Simon, \& Brener, 2000; Flechner, 2000; Turecki, 1999).

Dessa forma, é necessário ter clareza sobre os aspectos que podem ser patológicos na adolescência, estando relacionado, muitas vezes, com a intensidade, o grau e o desajuste com que aparecem as características próprias desta fase do desenvolvimento humano. $\mathrm{O}$ que se sabe é que o jovem que pensa, ameaça, tenta ou concretiza o suicídio está revelando, na verdade, um colapso em seus mecanismos adaptativos, de modo que tal situação é percebida não somente na adolescência, mas como em qualquer idade, como uma tentativa de alívio de sua dor e seu sofrimento.

Em termos de definição do construto ideação suicida, cabe mencionar que se refere ao pensamento ou idéia suicida. Engloba desejos, atitudes ou planos que o indivíduo tenha de se matar. Carlson e Cantwell (1982) comentam que a freqüência e a intensidade da ideação suicida aumentam com a idade cronológica, principalmente depois da puberdade. Neste sentido, um estudo realizado na Austrália com pacientes em internação psiquiátrica, mostrou que quase um quarto (22\%) dos pacientes com idades entre 15 e 24 anos apresentou ideação suicida (McKelvey, Pfaff, \& Acres, 2001).

Por outro lado, em um estudo com crianças e adolescentes da população geral dos Estados Unidos, foi possível estimar que de 7 a 12\% destes já tiveram uma ideação suicida séria (Maris et al., 2000). Evidenciou-se, ainda, em um estudo realizado com a população geral da cidade de Colúmbia (EUA), com 210 adolescentes, que 14\% apresentaram ideação suicida (Kashani, Goddard, \& Reid, 1989). Turvey, Stromquist, Kelly, Zwerling e Merchant (2002), realizando uma pesquisa na cidade de Iowa (EUA), revelaram que dos 1.617 adolescentes pesquisados, 8,2\% com 18 anos ou mais, apresentaram ideação suicida.

Outras investigações com estudantes da população geral apontam a prevalência de ideação suicida. Especificamente, um estudo realizado na Cidade do México, por González-Forteza, Berenton-Gorn, Tello-Granados, Facio-Florez e Medina-Mora (1998), mostrou índice de 11,8\% de ideação suicida, utilizando como método um estudo transversal e analisando duas amostras, uma de 1.712 alunas do ensino médio e outra com 30 adolescentes pacientes hospitalizados por tentativa de suicídio (amostra clínica). Garrison, Addy, Jackson, McKeown e Waller (1991) salientam que 70\% dos adolescentes da amostra, estudantes da cidade de Colúmbia, apresentaram ideação suicida, em um estudo longitudinal sobre sintomatologia depressiva, depressão maior e ideação suicida. Field, Diego e Sanders (2001) demonstraram que 18\% dos estudantes investigados na cidade de Miami, com idade média de 17 anos, relataram pensar em se matar. Este foi um estudo com 88 adolescentes do ensino médio com o preenchimento de um questionário sobre ideação suicida e sua relação com outras variáveis. Ainda, em um estudo também transversal, Stewart, Lam, Betson e Chung (1999) encontraram uma porcentagem de $40 \%$ de ideação suicida em 996 adolescentes da cidade de Hong Kong com idades entre 14 e 19 anos.
A respeito de estudos de gênero e ideação suicida, Man (1999) demonstrou que, no período da adolescência, as mulheres apresentam maiores taxas de ideação suicida quando comparadas aos homens (quatro vezes mais para ideação suicida e três vezes mais para tentativa de suicídio). Uma explicação possível para este fenômeno é de que as meninas apresentam maiores índices de depressão (principalmente depressão moderada) que os meninos (2:1, respectivamente), o que acaba aumentando a ideação suicida e as tentativas de suicídio em mulheres (Allison, Roeger, Martin, \& Keeves, 2001; Edwards \& Holden, 2001; Esposito \& Clum, 2002; Goldman \& Beardslee, 1999; Hesket, Ding \& Jenkins, 2002; Reinherz et al., 1995; Stewart et al., 1999). Vários outros estudiosos sobre a temática do suicídio, tanto no que se refere à população geral quanto à população clínica, também estimam que a depressão e a desesperança são fatores importantes para a predição de ideação suicida em adolescentes (Beck, Brown, \& Steer, 1997; Beck, Steer, \& Grisham, 2000; Burge \& Lester, 2001; Edwards \& Holden, 2001; Esposito \& Clum, 2002; Feijó, 1994; Hendin, 1991; Hovey \& King, 1996; Joiner, Pfaff, \& Acres, 2002; Kumar \& Steer, 1995; McGee, Williams, \& Nada-Raja, 2001; Nugent \& Williams, 2001; Sampson \& Mrazek, 2001; Schotte \& Clum, 1982; Shaffer \& Pfeffer, 2001; Thompson, Moody, \& Eggert, 1994; Yang \& Clum, 1994).

Especificamente sobre a depressão maior, Hauenstein (2003) menciona que este diagnóstico é bastante comum na adolescência, aumentando os riscos de ideação suicida. Dessa forma, a ideação suicida, segundo Carlson e Cantwell (1982), pode servir como um parâmetro da severidade de depressão.

Estudos realizados na população geral de adolescentes referem que a depressão e a desesperança explicam uma grande proporção da variância de ideação suicida (Kumar \& Steer, 1995; Man, 1999; Stewart et al., 1999). Segundo o estudo de Kumar e Steer (1995), os escores totais obtidos através do Inventário de Depressão de Beck (BDI) e da Escala de Desesperança de Beck (BHS) explicam aproximadamente 52\% da variância da Escala de Ideação Suicida de Beck (BSI). Na mesma direção, o estudo de Stewart et al. (1999), já mencionado, demonstrou que os escores do Inventário de Depressão de Beck (BDI) explicam 33\% da variância de ideação suicida na adolescência.

Com base no exposto, várias pesquisas têm sido desenvolvidas com o objetivo de identificar fatores de risco ou potenciais suicidas em adolescentes, o que tem possibilitado a utilização de escalas, como a Escala de Ideação Suicida de Beck (BSI), para identificar a presença de ideação suicida e de planejamento de suicídio em indivíduos em processo de atendimento psiquiátrico ambulatorial ou psicoterapêutico, ou seja, em estudos realizados com populações clínicas.

Pensando na população geral de adolescentes, bem como considerando os altos índices de suicídio encontrados em Porto Alegre, o presente estudo torna-se relevante uma vez que teve como objetivo identificar a existência de ideação suicida em adolescentes da população não-clínica. A existência de ideação suicida pode ser considerada como um importante fator de risco para o suicídio efetivo, junto com a depressão e a desesperança. Além disso, o suicídio na adolescência, como em qualquer idade, é uma morte antecipada que pode ser evita- 
da por meio de ações preventivas na família, escola, meios de comunicação e na comunidade como um todo, procurando, assim, promover o desenvolvimento saudável desses jovens.

Dessa forma, o objetivo geral desse estudo foi identificar a presença de ideação suicida em adolescentes da população geral (não-clínica), da cidade de Porto Alegre, com idades entre 15 e 19 anos. Os objetivos específicos foram: caracterizar os adolescentes com e sem ideação suicida quanto à intensidade de depressão e desesperança; identificar, nos adolescentes com ideação suicida, o nível de associação entre ideação suicida e depressão e/ou desesperança; identificar, nos adolescentes com ideação suicida, qual(is) variável(is) foi(ram) encontrada(s) como mais associadas(s) à ideação suicida.

\section{Método}

O estudo foi quantitativo, do tipo transversal, de levantamento e associação entre variáveis. Para calcular o tamanho mínimo da amostra necessária para a realização deste estudo, utilizou-se o cálculo amostral para proporções em populações grandes (ou infinitas), considerando a maximização de variância da proporção devido ao desconhecimento da mesma. Desta forma, obteve-se um tamanho de amostra confiável para qualquer proporção. Para esse cálculo, considerou-se uma confiança de $95 \%$ e um erro de $5 \%$. Assim, participaram do estudo 526 adolescentes que freqüentavam instituições escolares públicas e privadas de Porto Alegre, de ensino fundamental e médio, com idades entre 15 e 19 anos, do sexo feminino e masculino (ver Tabela 1).

Para a identificação sócio-demográfica da amostra foi utilizada uma ficha de dados pessoais com o objetivo de caracterizar e descrever os sujeitos em estudo. Os outros instrumentos utilizados, em sua versão brasileira foram: Escala de Ideação Suicida de Beck (BSI), Inventário de Depressão de Beck (BDI) e Escala de Desesperança de Beck (BHS), todos validados para a população não-clínica de adolescentes (Cunha, 2001; Werlang, Borges, \& Fensterseifer, 2004).

Para o desenvolvimento deste estudo, foram realizados contatos com instituições escolares públicas e privadas (através de uma rede de conveniência) obtendo-se a autorização necessária para a participação dos adolescentes. Todos os adolescentes e seus responsáveis assinaram o termo de consentimento livre e esclarecido para a participação na pesquisa.

Previamente à administração dos instrumentos, foi encaminhada uma carta aos pais e/ou responsáveis pelo aluno com idades entre 15 e 17 anos (por serem menores de 18 anos), acompanhada de uma ficha de consentimento livre e esclarecido com o objetivo de explicar a natureza e relevância da pesquisa a ser desenvolvida e obter autorização voluntária dos pais e/ou responsáveis para a participação do adolescente.

Após a assinatura do consentimento dos pais e/ou responsáveis, cada adolescente também assinou seu próprio consentimento de aceitação para participar da pesquisa. Tendo havido a devida aceitação para participação do estudo, a administração dos instrumentos foi realizada na própria instituição de ensino do adolescente, durante o horário escolar. A aplicação foi coletiva, com duração de aproximadamente 50 min (um período de aula).
Nos casos em que houve algum indício marcante (nos escores da BSI, BDI e BHS) de problemática mais grave, foi procurado o psicólogo ou o profissional responsável pelo Serviço de Orientação Educacional (SOE) da instituição e sugerido algum tipo de encaminhamento e intervenção preventiva para a turma em que foi localizado o caso.

Para a análise dos dados, em relação às variáveis sóciodemográficas, foi feita uma análise descritiva (cálculos de freqüências). No que diz respeito à estatística inferencial, foram utilizados o teste do qui-quadrado para averiguar as associações existentes entre depressão/desesperança e ideação suicida e a regressão logística para avaliar, entre as diversas variáveis do estudo, qual(is) foi(ram) mais associada(s) à ideação suicida.

O projeto de pesquisa deste estudo foi analisado e aprovado pelo Comitê de Ética em Pesquisa da Pontifícia Universidade Católica do Rio Grande do Sul.

\section{Resultados}

Dos 526 adolescentes que constituíram a amostra (ver Tabela 1) 295 (56,1\%) eram do sexo feminino, 231 (43,9\%), do sexo masculino; 290 (55,1\%) estudavam em escola pública e 236 (44,9\%), em escola privada, sendo que 215 (40,9\%) estavam no segundo ano do ensino médio. A maioria era solteira (91,6\%) e morava com, pelo menos, um integrante da família nuclear (pai, mãe, irmãos). Em relação à presença de alguma doença física e/ ou psicológica, 91,8\% afirmaram não manifestar doença física e 96,8\% responderam não possuir doença psicológica.

Tabela 1

Distribuição de freqüências (absolutas e percentuais) dos adolescentes participantes $(N=526)$

\begin{tabular}{|c|c|c|}
\hline Variável & $\begin{array}{c}\text { Freqüência } \\
\text { absoluta }\end{array}$ & $\begin{array}{l}\text { Freqüência } \\
\text { percentual }\end{array}$ \\
\hline Sexo feminino & 295 & 56,1 \\
\hline Sexo masculino & 231 & 43,9 \\
\hline 15 anos & 206 & 39,2 \\
\hline 16 anos & 175 & 33,3 \\
\hline 17 anos & 81 & 15,4 \\
\hline 18 anos & 50 & 9,5 \\
\hline 19 anos & 14 & 2,7 \\
\hline Ensino fundamental & 97 & 18,5 \\
\hline $1^{0}$ ano ensino médio & 125 & 23,8 \\
\hline $2^{\underline{0}}$ ano ensino médio & 215 & 40,9 \\
\hline $3^{0}$ ano ensino médio & 89 & 16,9 \\
\hline Escola pública & 290 & 55,1 \\
\hline Escola privada & 236 & 44,9 \\
\hline Solteiro & 482 & 91,7 \\
\hline Casado & 14 & 2,7 \\
\hline Amigado & 16 & 3,0 \\
\hline Separado & 3 & 0,6 \\
\hline Outro & 11 & 2,1 \\
\hline Mora com família & 482 & 91,6 \\
\hline Mora sozinho & 44 & 8,4 \\
\hline Tem doença física & 43 & 8,2 \\
\hline Não tem doença física & 483 & 91,8 \\
\hline Tem doença psicológica & 16 & 3,2 \\
\hline Não tem doença psicológica & 510 & 96,8 \\
\hline
\end{tabular}


Quanto à presença de ideação suicida, na amostra estudada foi possível constatar que 188 (36\%) adolescentes apresentaram ideação suicida, ou seja, um pouco mais de um terço da amostra. Destes, 67,6\% eram do sexo feminino. A distribuição percentual dos adolescentes com ideação suicida, por sexo e idade, pode ser observada na Tabela 2.

Tabela 2

Distribuição dos adolescentes com ideação suicida por sexo $e$ idade $(n=188)$

\begin{tabular}{lrrrrrr}
\hline Idade & \multicolumn{2}{r}{ Sexo } & Masculino & Sexo & Feminino & \multicolumn{2}{c}{ Total } \\
\cline { 2 - 7 } & $\mathbf{f}$ & \% & f & \% & f & \% \\
\hline 15 anos & 35 & 57,3 & 46 & 36,2 & 81 & 43,1 \\
16 anos & 15 & 24,5 & 47 & 37,0 & 62 & 33,0 \\
17 anos & 6 & 9,8 & 18 & 14,1 & 24 & 12,8 \\
18 anos & 5 & 8,1 & 12 & 9,4 & 17 & 9,0 \\
19 anos & 0 & 0,0 & 4 & 3,1 & 4 & 2,1 \\
\hline Total & 61 & 100,0 & 127 & 100,0 & 188 & 100,0 \\
\hline
\end{tabular}

Conforme os dados da Tabela 3, vê-se a distribuição de freqüências e porcentagens dos adolescentes com e sem ideação suicida, pontuadas na BSI, em relação à intensidade de depressão registrada no BDI.
O teste do qui-quadrado $\left(\chi^{2}=148,143 ; p<0,001\right)$ permitiu verificar a associação entre a intensidade de depressão (mínima $=0-11$; leve $=12-19$; moderada $=20-35$; grave $=36-63$ ) e a presença ou não de ideação suicida, em nível estatisticamente significativo. Dessa forma, depressão leve, moderada e grave, nesta amostra, estão associadas à presença de ideação suicida, assim como a depressão mínima está associada à ausência de ideação suicida.

Conforme os dados da Tabela 4, pode-se visualizar a distribuição de freqüências e porcentagens dos adolescentes com e sem ideação suicida, pontuadas na BSI, no que se refere à intensidade de desesperança registrada na BHS.

O teste do qui-quadrado $\left(\chi^{2}=119,869 ; p<0,001\right)$ permitiu verificar a associação entre a intensidade de desesperança ( ínima = 0-4; leve =5-8; moderada $=9-13$; grave $=14-20$ ) e a presença ou não de ideação suicida, em nível estatisticamente significativo. Dessa forma, desesperança leve, moderada e grave, nesta amostra, estão associadas à presença de ideação suicida, assim como a desesperança mínima está associada à ausência de ideação suicida.

O resultado da análise de regressão logística (ver Tabela 5) foi significativo $(p<0,05)$ demonstrando que, neste estudo, um adolescente com pontuação para depressão no BDI tem uma razão de chance (ou odds ratio) de 9,3 vezes de desenvolver ideação suicida, quando comparado com um adolescente que não tem depressão. Em relação à desesperança, um adolescente com pontuação para desesperança na

Tabela 3

Distribuição dos adolescentes por intensidade de depressão e presença de ideação suicida $(N=526)$

\begin{tabular}{|c|c|c|c|c|c|c|}
\hline \multirow[t]{2}{*}{ Intensidade depressão - BDI } & \multicolumn{2}{|c|}{ Com ideação suicida } & \multicolumn{2}{|c|}{ Sem ideação suicida } & \multicolumn{2}{|c|}{ Total } \\
\hline & f & $\%$ & f & $\%$ & f & $\%$ \\
\hline Depressão mínima & 66 & 35,1 & 282 & 83,4 & 348 & 66,1 \\
\hline Depressão leve & 54 & 28,7 & 47 & 13,9 & 101 & 19,2 \\
\hline Depressão moderada & 59 & 31,3 & 9 & 2,6 & 68 & 12,9 \\
\hline Depressão grave & 9 & 4,7 & 0 & 0,0 & 9 & 1,7 \\
\hline Total & 188 & 100,0 & 338 & 100,0 & 526 & 100,0 \\
\hline
\end{tabular}

Tabela 4

Distribuição dos adolescentes por intensidade de desesperança e presença de ideação suicida $(N=526)$

\begin{tabular}{lccrrrr}
\hline \multirow{2}{*}{ Intensidade desesperança - BHS } & \multicolumn{2}{c}{ Com ideação suicida } & \multicolumn{2}{c}{ Sem ideação suicida } & \multicolumn{2}{c}{ Total } \\
\cline { 2 - 7 } & f & \multicolumn{1}{c}{$\%$} & f & \multicolumn{1}{c}{ \% } & \multicolumn{1}{c}{ f } & $\%$ \\
\hline Desesperança mínima & 66 & 35,1 & 266 & 78,6 & 332 & 63,1 \\
Desesperança leve & 68 & 36,1 & 63 & 18,6 & 131 & 24,9 \\
Desesperança moderada & 45 & 23,9 & 7 & 2,6 & 52 & 9,8 \\
Desesperança grave & 9 & 4,7 & 2 & 0,5 & 11 & 2,0 \\
\hline Total & 188 & 100,0 & 338 & 100,0 & 526 & 100,0 \\
\hline
\end{tabular}


BHS tem 6,8 vezes de razões de chance de ter ideação suicida, quando comparado com um adolescente sem pontuação para desesperança. O sexo feminino comparado com o masculino demonstrou 2,1 vezes de razão de chance de ter ideação suicida. E o adolescente que conhece algum amigo que tenha tentado se matar, pode apresentar ideação suicida com uma razão de chance de 2,0 vezes em comparação a um adolescente que não conhece um amigo tentador.

Tabela 5

Resumo dos resultados da análise de regressão logística $(N=526)$

\begin{tabular}{lrrrcc}
\hline \multicolumn{1}{c}{ Variável } & $\begin{array}{c}\text { Coeficiente } \\
\text { de regressão }\end{array}$ & $\boldsymbol{t}$ & $\boldsymbol{p}$ & $\begin{array}{c}\text { Razão de } \\
\text { chance }\end{array}$ \\
\hline Sexo feminino & $-0,706$ & 8,604 & 0,003 & 2,1 \\
Depressão (BDI) & $-0,159$ & 55,801 & 0,000 & 9,3 \\
Desesperança (BHS) & $-0,147$ & 9,980 & 0,002 & 6,8 \\
Tentativa de amigo & $-0,689$ & 8,680 & 0,003 & 2,0 \\
Constante & 3,633 & & & \\
\hline
\end{tabular}

Ainda, considera-se importante mencionar que dos 188 adolescentes com ideação suicida, 100\% conheciam alguém que tinha tentado o suicídio e $64,4 \%$ conheciam alguém que tenha se suicidado. As porcentagens encontradas para o conhecimento de alguém que tenha tentado suicídio foram: 76\% conheciam amigo, 4\% um integrante da família nuclear (pai, mãe e irmãos), 12\% um parente de primeiro grau (avós, tios e primos) e $8 \%$ um vizinho. Para o conhecimento de alguém que tenha cometido o suicídio, as porcentagens encontradas foram: $69 \%$ conheciam um amigo, $18 \%$ vizinho e $13 \%$ um parente de primeiro grau (avós, tios e primos).

Em relação aos adolescentes sem ideação suicida (n = 338), observou-se que dos 338 61,9\% conheciam alguém que tinha tentado o suicídio e 50,1\% conheciam alguém que tinha cometido o suicídio. As porcentagens encontradas no que se refere ao conhecimento de alguém que tinha tentado o suicídio foram: 77\% conheciam um amigo, 10\% vizinho, $11 \%$ um parente de primeiro grau (avós, tios e primos) e $2 \%$ um integrante da família nuclear (pai, mãe e irmãos). Ainda, para as porcentagens encontradas sobre o conhecimento de alguém que tinha se suicidado foram: $74 \%$ conheciam um amigo, $17 \%$ vizinho, $7 \%$ um parente em primeiro grau (tios e primos) e $1 \%$ pai.

\section{Discussão}

A partir deste estudo, quando se têm dados de que 36\% dos adolescentes, de um total de 526 pesquisados, apresentaram ideação suicida, torna-se necessário pensar na intensidade e abrangência da problemática que se está enfocando. Esses jovens, principalmente em se tratando de uma população não-clínica, podem, portanto, estar expressando algo que vai além das características próprias da adolescência, ou seja, podem estar demonstrando um importante sofrimento decor- rente de um conflito interno, vislumbrando a possibilidade de morte como alternativa.

No que se refere a estudos internacionais de ideação suicida em adolescentes da população geral, pode-se comparar este dado (36\% com ideação suicida) com alguns estudos anteriores que demonstraram que de 7 a $14 \%$ da população adolescente já teve uma ideação suicida na vida (Kashani et al. 1989; Maris et al., 2000). Dessa forma, 36\% podem ser considerados um alto índice de ideação suicida encontrado na amostra estudada.

Em relação a estudos de adolescentes estudantes da população geral com ideação suicida, o resultado encontrado (36\%) pode ser comparado ao dado de 18\% (Field et al., 2001) de presença de ideação suicida em adolescentes do ensino médio com idade média de 17 anos e 40\% relacionado ao estudo de Stewart et al. (1999). Desse modo, 36\% é um índice alto, mas que constitui um resultado percentualmente similar ao estudo de Stewart et al. (1999), já mencionado, levando em conta as devidas proporções em estudos realizados em contextos diferenciados.

Especificamente em relação aos adolescentes com ideação suicida do presente estudo, 67,6\% eram do sexo feminino e $32,4 \%$ do sexo masculino, demonstrando dados que vão ao encontro de estudos mencionados anteriormente e que salientam que, na adolescência, as mulheres apresentam as maiores taxas de ideação suicida (quatro vezes mais) quando comparadas aos homens (Allison et al. 2001; Edwards \& Holden, 2001; Espósito \& Clum, 2002; Goldman \& Beardslee, 1999; Hesket et al. 2002; Man, 1999; Reinherz et al. 1995; Stewart et al. 1999).

Quanto às faixas etárias pesquisadas, entre os adolescentes com ideação suicida, a idade de 15 anos foi a mais freqüente. Comparando com a literatura, tal achado vai ao encontro do que é exposto no estudo de Reinherz et al. (1995), ou seja, 15 anos é uma idade considerada crítica para a manifestação de comportamento suicida na adolescência e, portanto, apresentar ideação suicida nesta idade torna-se preocupante.

A respeito do fato de conhecer alguém que tenha tentado ou cometido o suicídio, tanto entre os adolescentes com ideação suicida ( $n=188$ ) como os sem ideação suicida ( $n=$ 338), deve-se considerar dados da literatura que abordam o tema do suicídio contagioso (Gould et al. 1988; Stone, 1999), ou seja, um tipo de suicídio que leva em conta a vulnerabilidade do adolescente para identificar-se com um amigo, conhecido ou familiar que tenha tentado ou cometido suicídio. Em relação aos achados deste estudo, uma importante porcentagem dos adolescentes com ideação suicida conhecia um amigo que tinha tentado ou cometido suicídio, podendo-se pensar neste fato como um possível e importante fator de risco para o aparecimento de ideação suicida nestes jovens (Shaffer \& Pfeffer, 2001; WHO, 2001). Contudo, é importante ressaltar que os adolescentes sem ideação suicida também apresentaram uma alta porcentagem em conhecer alguém que tenha tentado ou cometido suicídio. Pode-se pensar, então, que este fator de risco, nesta amostra, não é o único preditor para 
o aparecimento de ideação suicida, ou seja, neste estudo há outras variáveis também influentes para a presença de ideação suicida, tais como a depressão e a desesperança. De qualquer forma, o fato de que de um total de 526 adolescentes, $82,8 \%$ conhecia alguém que tinha tentado e cometido o suicídio, aponta para a relevância de se pensar que esta temática está muito mais presente na vida das pessoas do que, de uma maneira geral, se pensa.

Respondendo aos objetivos específicos deste estudo, os dados demonstram, de forma similar aos achados da literatura internacional, que tanto a depressão quanto a desesperança estão associadas com a presença de ideação suicida, principalmente quando se fala de depressão e desesperança leve, moderada e grave (Beck et al., 1997; Beck et al., 2000; Burge \& Lester, 2001; Edwards \& Holden, 2001; Esposito \& Clum, 2002; Feijó, 1994; Hendin, 1991; Hovey \& King, 1996; Joiner et al., 2002; Kumar \& Steer, 1995; Maris et al., 2000; McGee et al., 2001; Nugent \& Williams, 2001; Sampson \& Mrazek, 2001; Schotte \& Clum, 1982; Shaffer \& Pfeffer, 2001; Thompson et al., 1994; Yang \& Clum, 1994). Além dessa associação existente, pode-se perceber que são, também, importantes variáveis associadas à ideação suicida, podendo significar que, neste estudo, adolescentes com depressão e/ou desesperança leve, moderada e grave podem desenvolver ideação suicida.

Uma vez que indivíduos que chegam a consumar o suicídio não podem ser tratados, considerável empenho tem sido realizado no sentido de identificar fatores de risco para tal comportamento. Assim, as investigações têm possibilitado estabelecer que, entre os fatores que contribuem para o comportamento suicida, têm um peso primordial os comprometimentos psicológicos. Dentre eles, a depressão é a que com mais freqüência se associa ao suicídio, seja como diagnóstico e/ou como sintoma. As pessoas com doença ou sintoma depressivo expressam freqüentemente o desejo de morrer, tentando, muitas vezes, se matar, até chegando realmente a cometer o suicídio. Além disso, o suicídio pode se tornar a única solução possível quando estão presentes expectativas negativas frente ao futuro. Desesperança, pois, representa um nexo causal entre depressão e suicídio (Cunha, 2001).

Desta forma, este fato mencionado se confirma na amostra deste estudo, sendo constatado como algo muito preocupante, uma vez que se têm, através da literatura, achados que demonstraram que há numerosos casos de adolescentes com depressão, desesperança e idéias de morte, que pensam ser capaz de, sem ajuda, resolver seus problemas (Culp, Clyman, \& Culp, 1995). Sabe-se que, quando tais sentimentos e comportamentos aparecem nos adolescentes há, na verdade, um pedido de ajuda e a representação de um sofrimento intenso.

Em relação às variáveis mais associadas à ideação suicida, pode-se observar o sexo feminino, o fato de se ter um amigo que tenha tentado o suicídio e características de depressão e desesperança. A partir desses dados, pode-se dizer que, dos 526 adolescentes pesquisados, os que eram do sexo feminino, que tinham um amigo que tinha tentado o suicídio e/ou que possuíam características de depressão e desesperança desenvolveram ideação suicida mais facilmente, quando comparados com os outros adolescentes.

Cabe salientar, que o resultado de $64 \%$ dos adolescentes sem ideação suicida era esperado, uma vez que se está trabalhando com uma população não-clínica. Pensando em nossa realidade brasileira, o resultado da presença de ideação suicida nestes 188 adolescentes faz pensar na confirmação, ou melhor, na relação que inclui Porto Alegre como sendo uma das capitais com maior índice de suicídio em adolescentes de 15 a 19 anos da idade.

Pensando-se em uma perspectiva preventiva, torna-se essencial identificar adolescentes com ideação suicida, já que este pode ser o primeiro passo para o suicídio, principalmente, quando associada a aspectos de depressão e desesperança. Desta forma, destaca-se a importância de programas de prevenção que evitem que as pessoas desenvolvam comportamentos suicidas ou adotem condutas autodestrutivas.

\section{Referências}

Allison, S., Roeger, L., Martin, G., \& Keeves, J. (2001). Gender differences in the relationship between depression and suicidal ideation in young adolescents. Australian and New Zealand Journal of Psychiatry, 35, 498-503.

Barrios, L. C., Everett, S. A., Simon, T. R., \& Brener, N. D. (2000). Suicide ideation among US college students: associations with other injury risk behaviors. Journal of American College Health, 48, 229-233.

Beck, A. T., Brown, G. K., \& Steer, R. A. (1997). Psychometric characteristics of the Scale for Suicide Ideation with psychiatric outpatients. Behavior Research and Therapy, 35(11), 1039-1046.

Beck, A T., Steer, R. A., \& Grisham, J. R. (2000). Risk factors for suicide in psychiatric outpatients: a 20-year prospective study. Journal of Consulting and Clinical Psychology, 3(68), 371-377.

Bertolote, J. M., \& Fleischmann, A. (2004). Suicídio e doença mental: uma perspectiva global. In N. J. Botega \& B. G. Werlang (Orgs.), Comportamento suicida (pp. 35-44). Porto Alegre: ArtMed.

Burge, M., \& Lester, D. (2001). Predicting suicidal ideation in high school students. Psychological Reports, 89, 283-284.

Carlson, G. A., \& Cantwell, D. P. (1982). Suicidal behavior and depression in children and adolescents. American Academy of Child Psychiatry, 21(4), 361-368.

Culp, A. M., Clyman, M. M., \& Culp, R. E. (1995). Adolescent depressed mood, reports of suicide attempts, and asking for help. Adolescence, 30(120), 827-837.

Cunha, J. A. (2001). Manual da versão em português das Escalas Beck. São Paulo: Casa do Psicólogo.

De Leo, D. (2004). Prefácio. In N. J. Botega \& B. G. Werlang (Orgs.), Comportamento suicida (pp. 13-14). Porto Alegre: ArtMed.

De Leo, D., Bertolote, J., \& Lester, D. (2003). La violencia autoinfligida. In E. G. Krug, L. L. Dahlberg, J. A. Mercy, A. B. Zuvi, \& P. R. Lozano (Orgs.), Informe Mundial de la violencia e de la salud (pp. 200-231). Washington: Organización Panamericana de la Salud.

Edwards, M. J., \& Holden, R. R. (2001). Coping, meaning in life and suicidal manifestations examining gender differences. Journal of Clinical Psychology, 57(12), 1517-1534

Esposito, C. L., \& Clum, G. A. (2002). Psychiatric symptoms and their relationship to suicidal ideation in a high-risk adolescent community sample. Journal of American Academic Child and Adolescent Psychiatry, 41(1), 44-51. 
Feijó, R. B. (1994). Repercussão da presença de doença orgânica e da suspeita de transtorno mental através de escalas de sintomatologia depressiva de risco de suicídio e de expectativa de futuro. Tese de Doutorado não-publicada, Universidade Federal do Rio Grande do Sul, Porto Alegre.

Field, T., Diego, M., \& Sanders, C. E. (2001). Adolescent suicidal ideation. Adolescence, 36(142), 241-248.

Flechner, S. (2000). Psicoanálisis y cultura: la clínica actual de pacientes adolescentes em riesgo. Um nuevo desafío? Revista Latino-Americana de Psicanálise, (4), 467-482.

Garrison, C. Z., Addy, C. L., Jackson, K. L., McKeown, R. E., \& Waller, J. L. (1991). A longitudinal study of suicidal ideation in young adolescents. Journal of American Academic Child and Adolescent Psychiatry, 30(4), 597-603.

Goldman, S., \& Beardslee, W. R. (1999). Suicide in children and adolescents. In D. G. Jacobs (Org.), Suicide assessment and intervention (pp. 417-442). San Francisco: Jossey-Bass.

González-Forteza, C., Berenzon-Gorn, S., Tello-Granados, A. M., Facio-Florez, D., \& Medina-Mora Icaza, M. E. (1998). Suicidal ideation and associated characteristics in adolescent women. Salud Pública de México, 40(5), 430-437.

Gould, M. S., Wallenstein, S., \& Davidson, L. (1989). Suicide clusters: a critical review. In I. S. Lann, E. K. Móscicki, \& R. W. Maris (Orgs.), Strategies for studying suicide and suicidal behavior (pp. 17-29). Nova York: Guilford.

Hauenstein, E. J. (2003). Depression in adolescence. Journal of Obstetric Gynecologic Neonatal Nursing, 32(2), 239-248.

Hendin, H. (1991). Psychodynamics of suicide, with particular reference to the young. American Journal Psychiatry, 148(9), 1150-1158.

Hesket, T., Ding, Q. J., \& Jenkins, R. (2002). Suicide ideation in Chinese adolescents. Social Psychiatry and Psychiatric Epidemiology, 37(5), 230-235.

Hovey, J. D., \& King, C. A. (1996). Acculturative stress, depression, and suicidal ideation among immigrant and second-generation Latino adolescents. Journal of American Academic Child and Adolescent Psychiatry, 35(9), 1183-1192.

Joiner, T. E. Jr., Pfaff, J. J., \& Acres, J. G. (2002). Characteristics of suicidal adolescents and young adults presenting primary care with non-suicidal (indeed non-psychological) complaints. European Journal of Public Health, 12(3), 177-179.

Kashani, J. H., Goddard, P., \& Reid, J. C. (1989). Correlates of suicide ideation in a community sample of children and adolescents. Journal of American Academy of Child and Adolescent Psychiatry, 28(6), 912-917.

Kumar, G. \& Steer, R. A. (1995). Psychosocial correlates of suicidal ideation in adolescent psychiatric inpatients. Suicide and Life Threatening Behavior, 25(3), 339-346.

Man, A. F. (1999). Correlates of suicide ideation in high school students: the importance of depression. The Journal of Genetic Psychology, 160(1), 105-114.

Mann, J. J. (2002). A current perspective of suicide and attempted suicide. Annual International Medicine, 136, 302-311.

Maris, R. W., Berman, L., \& Silverman, M. M. (2000). Comprehensive textbook of suicidology. Nova York: Guilford.
McGee, R., Williams, S., \& Nada-Raja, S. (2001). Low self-esteem and hopelessness in childhood and suicidal ideation in early adulthood. Journal of Abnormal Child Psychology, 29(4), 281-291.

McKelvey, R. S., Pfaff, J. J., \& Acres, J. G. (2001). The relationship between chief complaints, psychological distress, a suicidal ideation in 15-24-yearold patients presenting to general practitioners. Medical Journal of Australia, 175(10), 550-552.

Nugent, W. R., \& Williams, M. (2001). The relationship between the comorbidity of depression with problems in psychosocial functioning and the severity of suicidal ideation. The Social Service Review, 75(4), 581-604.

Reinherz, H. Z., Giaconia, R. M., Silverman, A. B., Friedman, A., Parkis, B., Cohen, E. et al. (1995). Early psychosocial risks for adolescent suicidal ideation and attempts. Journal of American Academic Child and Adolescent Psychiatry, 34(5), 599-611.

Sampson, S. M., \& Mrazek, D. A. (2001). Depression in adolescence. Current Opinion in Pediatrics, 13(6), 586-590.

Schotte, D. E., \& Clum, G. A. (1982). Suicide ideation in a college population: a test of a model. Journal of Consulting and Clinical Psychology, 50(5), 690-696.

Shaffer, D., \& Pfeffer, C. R. (2001). Practice parameter for the assessment and treatment of children and adolescents with suicidal behavior. Journal of American Academic Child and Adolescent Psychiatry, 40(7), 24-51.

Souza, E. R., Minayo, M. C. S., \& Malaquias, J. V. (2002). Suicide among young people in selected Brazilian state capitals. Cadernos de Saúde Pública, 18(3), 673-683.

Stewart, S. M., Lam, T. H., Betson, C., \& Chung, S. F. (1999). Suicide ideation and its relationship to depressed mood in a community sample of adolescents in Hong Kong. Suicide and Life Threatening Behavior, 29(3), 227-240.

Stone, G. (1999). Suicide and attempt suicide. Nova York: Carroll \& Graf.

Thompson, E. A., Moody, K. A., \& Eggert, L. L. (1994.) Discriminating suicide ideation among high-risk youth. Journal of School Health, 64(9), 361-367.

Turecki, G. (1999). O suicídio e sua relação com o comportamento impulsivoagressivo. Revista Brasileira de Psiquiatria, 21(2), 18-22.

Turvey, C., Stromquist, A., Kelly, K., Zwerling, C., \& Merchant, J. (2002). Financial loss and suicidal ideation in a rural community sample. Acta Psychiatrica Scandinavica, 106(5), 373-380.

Werlang, B. S. G., Borges, V. R., \& Fensterseifer, L. (2004). Estudo de fidedignidade e validade da Escala de Ideação Suicida de Beck. In B. S. G. Werlang \& N. J. Botega (Orgs), Comportamento suicida (pp. 189-193). Porto Alegre: Artmed.

World Health Organization - WHO. (2001). Prevención del suicidio: un instrumento para docentes y demás personal institucional. Obtido de http:// www.who.int/mental_health/.

Yang, B., \& Clum, G. A. (1994). Life stress, social support, and problem-solving skills predictive of depressive symptoms, hopelessness, and suicide ideation in an Asian student population: a test of a model. Suicide and Life Threatening Behavior, 24(2), 127-139.

Vivian Roxo Borges, mestre em Psicologia Clínica pela Pontifícia Universidade Católica do Rio Grande do Sul, é professora no Curso de Psicologia na Universidade Regional Integrada do Alto Uruguai e das Missões Campus de Erechim, e Coordenadora do Curso de Pedagogia da Instituição Educacional São Judas Tadeu. Endereço para correspondência: Rua Carlos Trein Filho, 870, apt. 802; Porto Alegre, RS; CEP 90450-120. Tel.: (51) 3023-5285. E-mail: viviborg@terra.com.br

Blanca Susana Guevara Werlang, doutora em Ciências Médicas na área de Saúde Mental pela Universidade Estadual de Campinas, é Professora Adjunta no Programa de Pós-Graduação em Psicologia e Diretora da Faculdade de Psicologia. Pontifícia Universidade Católica do Rio Grande do Sul. E-mail: bwerlang@pucrs.br 